\title{
LIGHTLIKE SUBMANIFOLDS OF AN INDEFINITE SASAKIAN MANIFOLD WITH A NON-METRIC $\theta$-CONNECTION
}

\author{
DAE HO JIN
}

\begin{abstract}
In this paper, we study two types of 1-lightlike submanifolds, named by lightlike hypersurface and half lightlike submanifold, of an indefinite Sasakian manifold admitting non-metric $\theta$-connections. We prove that there exist no such two types of 1-lightlike submanifolds of an indefinite Sasakian manifold.
\end{abstract}

\section{INTRODUCTION}

A linear(affine) connection $\bar{\nabla}$ on a semi-Riemannian manifold $(\bar{M}, \bar{g})$ is called a non-metric $\theta$-connection if, for any vector fields $X, Y$ and $Z$ on $\bar{M}$, it satisfies

$$
\left(\bar{\nabla}_{X} \bar{g}\right)(Y, Z)=-\theta(Y) \bar{g}(X, Z)-\theta(Z) \bar{g}(X, Y),
$$

where $\theta$ is a 1 -form, associated with a non-vanishing smooth vector field $\zeta$ by

$$
\theta(X)=\bar{g}(X, \zeta)
$$

Two special cases are important for both the mathematical study and the applications to physics: (1) A non-metric $\theta$-connection $\bar{\nabla}$ on $\bar{M}$ is called a semi-symmetric non-metric connection if its torsion tensor $\bar{T}$ satisfies

$$
\bar{T}(X, Y)=\theta(Y) X-\theta(X) Y .
$$

The notion of semi-symmetric non-metric connections on a Riemannian manifold was introduced by Ageshe and Chafle [1] and later studied by many authors. The lightlike version of Riemannian manifolds with semi-symmetric non-metric connections have been studied by some authors $[11,12,13,14,17]$.

(2) A non-metric $\theta$-connection $\bar{\nabla}$ is called a quarter-symmetric non-connection if its torsion tensor $\bar{T}$ satisfies

$$
\bar{T}(X, Y)=\theta(Y) \phi X-\theta(X) \phi Y,
$$

Received by the editors January 14, 2014. Accepted September 24, 2014.

2010 Mathematics Subject Classification. 53C25, 53C40, 53C50.

Key words and phrases. non-metric $\theta$-connections, lightlike hypersurface, half lightlike submanifold. 
where $\phi$ is a $(1,1)$-type tensor field. The quarter-symmetric non-metric connection was introduced by S. Golad [8], and then, studied by many authors [2, 3, 16].

The theory of lightlike submanifolds is an important topic of research in differential geometry due to its application in mathematical physics. The study of such notion was initiated by Duggal and Bejancu [4] and later studied by many authors $[6,7]$. Although now we have lightlike version of a large variety of Riemannian submanifolds, the geometry of lightlike submanifolds of indefinite Sasakian manifolds admitting non-metric $\theta$-connections has not been introduced as yet.

In this paper, we study the geometry of two type of lightlike submanifolds, named by lightlike hypersurface and half lightlike submanifold, of an indefinite Sasakian manifold $\bar{M}$ admitting non-metric $\theta$-connection, in which the 1 -form $\theta$ and its associated vector field $\zeta$, defined by (1.1), is identical with the structure 1 -form $\theta$ and its associated vector field $\zeta$, respectively, of the indefinite Sasakian structure $(J, \zeta, \theta, \bar{g})$. We prove the following result:

- There exist no such two types of 1-lightlike submanifolds of an indefinite Sasakian manifold $\bar{M}$ admitting non-metric $\theta$-connections.

From these results we deduce to the following result:

- There exist no such two types of 1-lightlike submanifolds of an indefinite Sasakian manifold $\bar{M}$ admitting either semi-symmetric non-metric connection or quartersymmetric non-metric connection.

\section{Non-existence Theorem for Lightlike Hypersurfaces}

An odd-dimensional semi-Riemannian manifold $(\bar{M}, \bar{g})$ is said to be an indefinite Sasakian manifold $([9] \sim[10])$ if there exists a structure set $\{J, \zeta, \theta, \bar{g}\}$, where $J$ is a $(1,1)$-type tensor field, $\zeta$ is a vector field which is called the structure vector field of $\bar{M}$ and $\theta$ is a 1 -form such that, for any vector fields $X$ and $Y$ on $\bar{M}$,

$$
\begin{gathered}
J^{2} X=-X+\theta(X) \zeta, \bar{g}(J X, J Y)=\bar{g}(X, Y)-\epsilon \theta(X) \theta(Y), \theta(\zeta)=1, \\
\left(\bar{\nabla}_{X} J\right) Y=\bar{g}(X, Y) \zeta-\epsilon \theta(Y) X
\end{gathered}
$$

holds, where $\epsilon=1$ or -1 according as $\zeta$ is spacelike or timelike respectively.

In this case, we show that $J \zeta=0$ and $\theta \circ J=0$. The structure set $\{J, \zeta, \theta, \bar{g}\}$ is called an indefinite Sasakian structure of $\bar{M}$. From (2.1) and (2.2), we get

$$
\bar{\nabla}_{X} \zeta=-\epsilon J X, \quad d \theta(X, Y)=\bar{g}(X, J Y) .
$$

In the entire discussion of this article, we shall assume that the structure vector field $\zeta$ of $\bar{M}$ to be unit spacelike, i.e., $\epsilon=1$, without loss of generality. 
Let $(M, g)$ be a lightlike hypersurface, with a screen distribution $S(T M)$, of an indefinite Sasakian manifold $(\bar{M}, \bar{g})$. We follow Duggal and Bejancu [4] for notations and structure equations used in this section. For any null section $\xi$ of $T M^{\perp}$ on a coordinate neighborhood $\mathcal{U} \subset M$, there exists a unique null section $N$ of a unique vector bundle $\operatorname{tr}(T M)$ in $S(T M)^{\perp}$ satisfying

$$
\bar{g}(\xi, N)=1, \quad \bar{g}(N, N)=\bar{g}(N, X)=0, \quad \forall X \in \Gamma(S(T M)) .
$$

We call $\operatorname{tr}(T M)$ and $N$ the transversal vector bundle and the null transversal vector field of $M$ with respect to the screen distribution respectively. Let $P$ be the projection morphism of $T M$ on $S(T M)$. Then the local Gauss and Weingartan formulas of $M$ and $S(T M)$ are given respectively by

$$
\begin{aligned}
& \bar{\nabla}_{X} Y=\nabla_{X} Y+B(X, Y) N \\
& \bar{\nabla}_{X} N=-A_{N} X+\tau(X) N \\
& \nabla_{X} P Y=\nabla_{X}^{*} P Y+C(X, P Y) \xi \\
& \nabla_{X} \xi=-A_{\xi}^{*} X-\sigma(X) \xi
\end{aligned}
$$

for any $X, Y \in \Gamma(T M)$, where the symbols $\nabla$ and $\nabla^{*}$ are the induced linear connections on $T M$ and $S(T M)$ respectively, $B$ and $C$ are the local second fundamental forms on $T M$ and $S(T M)$ respectively, $A_{N}$ and $A_{\xi}^{*}$ are the shape operators on $T M$ and $S(T M)$ respectively and $\tau$ and $\sigma$ are 1-forms on $T M$.

The induced connection $\nabla$ on $M$ is not metric and satisfies

$$
\begin{aligned}
\left(\nabla_{X} g\right)(Y, Z)= & B(X, Y) \eta(Z)+B(X, Z) \eta(Y) \\
& -\theta(Y) g(X, Z)-\theta(Z) g(X, Y)
\end{aligned}
$$

for any $X, Y, Z \in \Gamma(T M)$, where $\eta$ is a 1 -form on $T M$ such that

$$
\eta(X)=\bar{g}(X, N), \quad \forall X \in \Gamma(T M) .
$$

From the fact that $B(X, Y)=\bar{g}\left(\bar{\nabla}_{X} Y, \xi\right)$, we know that $B$ is independent of the choice of the screen distribution $S(T M)$, and satisfies

$$
B(X, \xi)=0, \quad \forall X \in \Gamma(T M) .
$$

From this result and (2.4), for all $X \in \Gamma(T M)$ we obtain

$$
\bar{\nabla}_{X} \xi=-A_{\xi}^{*} X-\sigma(X) \xi .
$$


Now we set $a=\theta(N)$ and $b=\theta(\xi)$. For all $X, Y \in \Gamma(T M)$, the above second fundamental forms $B$ and $C$ are related to their shape operators by

$$
\begin{aligned}
& g\left(A_{\xi}^{*} X, Y\right)=B(X, Y)-b g(X, Y), \quad \bar{g}\left(A_{\xi}^{*} X, N\right)=0, \\
& g\left(A_{N} X, P Y\right)=C(X, P Y)-a g(X, P Y)-\eta(X) \theta(P Y), \\
& \bar{g}\left(A_{N} X, N\right)=-a \eta(X), \quad \sigma(X)=\tau(X)-b \eta(X) .
\end{aligned}
$$

Now we quote the following result by Jin [9]:

Lemma 1. Let $M$ be a lightlike hypersurface of an indefinite almost contact metric manifold $\bar{M}$. Then $J\left(T M^{\perp}\right)$ and $J(\operatorname{tr}(T M))$ are subbundles of $S(T M)$, of rank 1 .

Theorem 2.1. There exist no lightlike hypersurfaces of an indefinite Sasakian manifold admitting a non-metric $\theta$-connection.

Proof. Now we consider two vector fields $V$ and $U$ on $S(T M)$ such that

$$
J \xi=-V, \quad J N=-U .
$$

For any $X \in \Gamma(T M)$, the action $J X$ of $X$ by $J$ is expressed as

$$
J X=F X+u(X) N,
$$

where $F X$ is the tangential component of $J X$ and $u$ is a 1 -form given by

$$
u(X)=g(X, V), \quad \forall X \in \Gamma(T M) .
$$

Applying $\bar{\nabla}_{X}$ to $(2.13)_{1}$ and using $(2.2),(2.4),(2.10)$ and (2.14), we have

$$
\begin{aligned}
& \nabla_{X} V=F\left(A_{\xi}^{*} X\right)-\sigma(X) V+b X, \\
& B(X, V)=u\left(A_{\xi}^{*} X\right), \quad \forall X \in \Gamma(T M) .
\end{aligned}
$$

On the other hand, taking $Y=V$ to (2.11) and using (2.15), we have

$$
B(X, V)=u\left(A_{\xi}^{*} X\right)+b u(X), \quad \forall X \in \Gamma(T M) .
$$

From the last two equations, we obtain $b u(X)=0$ for all $X \in \Gamma(T M)$. Taking $X=V$ to this result and using (2.1), we get $b=0$. This implies that $\zeta$ is tangent to $M$. Replacing $Y$ by $\zeta$ to (2.4) and using (2.3), we obtain

$$
\nabla_{X} \zeta=-F X, \quad B(X, \zeta)=-u(X), \quad \forall X \in \Gamma(T M) .
$$

Applying $\nabla_{X}$ to $g(\zeta, \zeta)=1$ and using (2.8), we get

$$
g\left(\nabla_{X} \zeta, \zeta\right)=\theta(X)-a B(X, \zeta), \quad \forall X \in \Gamma(T M) .
$$

Substituting (2.16) $)_{1}$ into the last equation and using (2.15) and $(2.16)_{2}$, we have $\theta=0$ on $T M$. It is a contradiction as $\theta(\zeta)=1$. Thus there exist no lightlike hypersurfaces of an indefinite Sasakian manifold admitting a non-metric $\theta$-connection. 
Corollary 1. There exist no lightlike hypersurfaces of an indefinite Sasakian manifold admitting a semi-symmetric non-metric connection or a quarter-symmetric nonmetric connection.

\section{Non-existence Theorem for Half Lightlike Submanifolds}

Let $(M, g)$ be a half lightlike submanifold, with a screen distribution $S(T M)$ and the radical distribution $\operatorname{Rad}(T M)$, of an indefinite Sasakian manifold $(\bar{M}, \bar{g})$. We follow Duggal and Jin [5] for notations and structure equations used in this section. For any null section $\xi$ of $\operatorname{Rad}(T M)$, there exists a uniquely defined lightlike vector bundle $\operatorname{lt}(T M)$ and a null vector field $N$ of $\operatorname{lt}(T M)$ satisfying

$$
\bar{g}(\xi, N)=1, \quad \bar{g}(N, N)=\bar{g}(N, X)=\bar{g}(N, L)=0, \quad \forall X \in \Gamma(S(T M)) .
$$

We call $N, \operatorname{lt}(T M)$ and $\operatorname{tr}(T M)=S\left(T M^{\perp}\right) \oplus_{\text {orth }} \operatorname{ltr}(T M)$ the lightlike transversal vector field, lightlike transversal vector bundle and transversal vector bundle of $M$ with respect to $S(T M)$ respectively. In this case, the local Gauss and Weingartan formulas of $M$ and $S(T M)$ are given by

$$
\begin{aligned}
& \bar{\nabla}_{X} Y=\nabla_{X} Y+B(X, Y) N+D(X, Y) L, \\
& \bar{\nabla}_{X} N=-A_{N} X+\tau(X) N+\rho(X) L, \\
& \bar{\nabla}_{X} L=-A_{L} X+\phi(X) N, \\
& \nabla_{X} P Y=\nabla_{X}^{*} P Y+C(X, P Y) \xi, \\
& \nabla_{X} \xi=-A_{\xi}^{*} X-\sigma(X) \xi,
\end{aligned}
$$

for all $X, Y \in \Gamma(T M)$, where $\nabla$ and $\nabla^{*}$ are induced linear connections on $T M$ and $S(T M)$ respectively, $B$ and $D$ are called the local second fundamental forms of $M$, $C$ is called the local second fundamental form on $S(T M) . A_{N}, A_{\xi}^{*}$ and $A_{L}$ are linear operators on $T M$ and $\tau, \rho, \phi$ and $\sigma$ are 1-forms on $T M$.

Using (1.1) and (3.1), for all $X, Y, Z \in \Gamma(T M)$ we have

$$
\begin{aligned}
\left(\nabla_{X} g\right)(Y, Z) & =B(X, Y) \eta(Z)+B(X, Z) \eta(Y) \\
& -\theta(Y) g(X, Z)-\theta(Z) g(X, Y) .
\end{aligned}
$$

From the facts $B(X, Y)=\bar{g}\left(\bar{\nabla}_{X} Y, \xi\right)$ and $D(X, Y)=\bar{g}\left(\bar{\nabla}_{X} Y, L\right)$, we know that $B$ and $D$ are independent of the choice of $S(T M)$ and satisfy

$$
B(X, \xi)=0, \quad D(X, \xi)=-\phi(X), \quad \forall X \in \Gamma(T M) .
$$

From this result and (3.1), for all $X \in \Gamma(T M)$ we obtain

$$
\bar{\nabla}_{X} \xi=-A_{\xi}^{*} X-\sigma(X) \xi-\phi(X) L .
$$


We set $b=\theta(\xi), a=\theta(N)$ and $e=\theta(L)$. For any $X, Y \in \Gamma(T M)$, the above three local second fundamental forms are related to their shape operators by

$$
\begin{aligned}
& g\left(A_{\xi}^{*} X, Y\right)=B(X, Y)-b g(X, Y), \quad \bar{g}\left(A_{\xi}^{*} X, N\right)=0, \\
& g\left(A_{L} X, Y\right)=D(X, Y)-e g(X, Y)+\phi(X) \eta(Y), \\
& \bar{g}\left(A_{L} X, N\right)=\rho(X)-e \eta(X), \\
& g\left(A_{N} X, P Y\right)=C(X, P Y)-a g(X, P Y)-\eta(X) \theta(P Y), \\
& \bar{g}\left(A_{N} X, N\right)=-a \eta(X), \quad \sigma(X)=\tau(X)-b \eta(X) .
\end{aligned}
$$

Now we quote the following result by Jin [10]:

Lemma 2. Let $M$ be a half lightlike submanifold of an indefinite almost contact metric manifold $\bar{M}$. Then the distributions $J\left(T M^{\perp}\right), J(\operatorname{tr}(T M))$ and $J\left(S\left(T M^{\perp}\right)\right)$ are vector subbundles of $S(T M)$, of rank 1 .

Theorem 3.1. There exist no half lightlike submanifolds of an indefinite Sasakian manifold admitting a non-metric $\theta$-connection.

Proof. Now we consider three vector fields $V, U$ and $W$ on $S(T M)$ such that

$$
J \xi=-V, \quad J N=-U, \quad W=-J L .
$$

For any $X \in \Gamma(T M)$, the action $J X$ of $X$ by $J$ is expressed as

$$
J X=F X+u(X) N+w(X) L,
$$

where $F X$ is the tangential component of $J X$ and $u$ and $w$ are 1 -forms given by

$$
u(X)=g(X, V) \quad w(X)=g(X, W), \quad \forall X \in \Gamma(T M) .
$$

Applying $\bar{\nabla}_{X}$ to $(3.12)_{1}$ and using (2.2), (3.1), (3.8) and (3.13), we have

$$
\begin{aligned}
& \nabla_{X} V=F\left(A_{\xi}^{*} X\right)-\sigma(X) V-\phi(X) W+b X, \\
& B(X, V)=u\left(A_{\xi}^{*} X\right), \quad D(X, V)=w\left(A_{\xi}^{*} X\right), \quad \forall X \in \Gamma(T M) .
\end{aligned}
$$

On the other hand, taking $Y=V$ to (3.9) and using (3.14) $)_{1}$, we have

$$
B(X, V)=u\left(A_{\xi}^{*} X\right)+b u(X) .
$$

From this and $(3.16)_{1}$, we obtain $b u(X)=0$ for any $X \in \Gamma(T M)$. Thus we get $b=0$. It follow that $B(X, Y)=g\left(A_{\xi}^{*} X, Y\right)$ and $\tau=\sigma$. Applying $\bar{\nabla}_{X}$ to $(3.12)_{3}$ and using (2.2), (3.1), (3.3), (3.12) and (3.13), we have

$$
\begin{aligned}
& \nabla_{X} W=F\left(A_{L} X\right)+\phi(X) U+e X, \\
& B(X, W)=u\left(A_{L} X\right), \quad D(X, W)=w\left(A_{L} X\right) .
\end{aligned}
$$


On the other hand, taking $Y=W$ to (3.10), we have

$$
D(X, W)=w\left(A_{L} X\right)+e w(X) .
$$

From this and $(3.18)_{2}$, we obtain $e w(X)=0$ for any $X \in \Gamma(T M)$. Thus we get $e=0$. As $b=e=0$, the structure vector field $\zeta$ is tangent to $M$.

Applying $\bar{\nabla}_{X}$ to (3.13) and using (3.1) (3.3), (3.12) and (3.13), we have

$$
\begin{aligned}
\left(\nabla_{X} F\right) Y & =u(Y) A_{N} X+w(Y) A_{L} X-B(X, Y) U-D(X, Y) W \\
& +g(X, Y) \zeta-\theta(Y) X \\
\left(\nabla_{X} u\right) Y & =-u(Y) \tau(X)-w(Y) \phi(X)-B(X, F Y) \\
\left(\nabla_{X} w\right)(Y) & =-u(Y) \rho(X)-D(X, F Y)
\end{aligned}
$$

On the other hand, applying $\nabla_{X}$ to $u(Y)=g(Y, V)$ and $w(Y)=g(Y, W)$ by turns and using (3.6), (3.15), (3.16) $1,(3.17),(3.18)_{1}$ and $\theta \circ J=0$, we have

$$
\begin{aligned}
& \left(\nabla_{X} u\right)(Y)=-u(Y) \tau(X)-w(Y) \phi(X)-B(X, F Y)-\theta(Y) u(X), \\
& \left(\nabla_{X} w\right)(Y)=-u(Y) \rho(X)-D(X, F Y)-\theta(Y) w(X) .
\end{aligned}
$$

From the last four equations, for all $X, Y \in \Gamma(T M)$ we obtain

$$
\theta(Y) u(X)=0, \quad \theta(Y) w(X)=0 .
$$

Taking $X=U$ and $Y=\zeta$ to the first equation, or taking $X=W$ and $Y=\zeta$ to the second equation, we have $1=0$. It is a contradiction. Thus there exist no half lightlike submanifolds of an indefinite Sasakian manifold admitting a non-metric $\theta$-connection.

Corollary 2. There exist no half lightlike submanifolds of an indefinite Sasakian manifold admitting a semi-symmetric non-metric connection or a quarter-symmetric non-metric connection.

\section{REFERENCES}

1. N.S. Ageshe \& M.R. Chafle: A semi-symmetric non-metric connection on a Riemannian manifold. Indian J. Pure Appl. Math. 23 (1992), no. 6, 399-409.

2. M. Ahmad \& A. Haseeb: Hypersurfaces of an almost $r$-paracontact Riemannian manifold endowed with a quarter-symmetric non-metric connection. Kyungpook Math. J. 49 (2009), 533-543.

3. A. Barman: On a type of quarter-symmetric non-metric $\phi$-connection on a Kenmotsu manifold. Bull. Math. Analy. and Appl. 4 (2012), no. 3, 1-11. 
4. K.L. Duggal \& A. Bejancu: Lightlike Submanifolds of Semi-Riemannian Manifolds and Applications. Kluwer Acad. Publishers, Dordrecht, 1996.

5. K.L. Duggal \& D.H. Jin: Half-lightlike submanifolds of codimension 2. Math. J. Toyama Univ. 22 (1999), 121-161.

6. : Null curves and Hypersurfaces of Semi-Riemannian Manifolds. World Scientific, 2007

7. K.L. Duggal \& B. Sahin: Differential geometry of lightlike submanifolds. Frontiers in Mathematics, Birkhäuser, 2010.

8. S. Golab: On semi-symmetric and quarter-symmetric connections. Tensor, N.S. 29 (1975), 249-254.

9. D.H. Jin: Geometry of lightlike hypersurfaces of an indefinite Sasakian manifold. Indian J. Pure and Appl. Math. 41 (2010), no. 4, 569-581.

10. __ Half lightlike submanifolds of an indefinite Sasakian manifold. J. Korean Soc Math. Edu. Ser. B: Pure Appl. Math. 18 (2011), no. 2, 173-183.

11. ___ Lightlike submanifolds of a semi-Riemannian manifold with a semi-symmetric non-metric connection. J. Korean Soc Math. Edu. Ser. B. 19 (2012), no. 3, 211-228.

12. ___ : Einstein lightlike hypersurfaces of a Lorentz space form with a semi-symmetric non-metric connection. Bull. Korean Math. Soc. 50 (2013), no. 4, 1367-1376.

13. ___ Einstein half lightlike submanifolds of a Lorentzian space form with a semisymmetric non-metric connection. J. Ineq. and Appl. (2013), 2013:403.

14. D.H. Jin \& J.W. Lee: A classification of half lightlike submanifolds of a semiRiemannian manifold with a semi-symmetric non-metric connection. Bull. Korean Math. Soc. 50 (2013), no. 3, 705-717.

15. S. Sasaki: Almost contact manifolds. Lecture notes, Math. Inst., Tôhoku Univ., Vol. I, II, III, 1965-1968.

16. S. Yadav \& D.L. Suthar: A quart symmetric non-metric connection in a generalized co-symmpletic manifolds. Glob. J. Sci. Fron. Res 11 (2011), no. 9, 1-7.

17. E. Yaşar, A.C. Çöken \& A. Yücesan: Lightlike hypersurfaces in semi-Riemannian manifold with semi-symmetric non-metric connection. Math. Scand. 102 (2008), 253264.

Department of Mathematics, Dongguk University, Gyeonguu 780-714, Republic of KoREA

Email address: jindh@dongguk.ac.kr 